\title{
Design and Implementation of UAV Flight Simulation Based on Matlab/Simulink
}

\author{
Zheng Xing ${ }^{1, \mathrm{a}}$, Yang $\mathrm{He}^{2, \mathrm{~b}}$, Cheng Jian ${ }^{1, \mathrm{c}}$ \\ ${ }^{1}$ Wuhan Mechanical Technology College, Wuhan, 430075, China \\ ${ }^{2}$ Hubei University of Education, Wuhan, 430205, China \\ aemail:zhengxcn@foxmail.com, bemail:yanghe_80@163.com, 'email:plachengjian_81@chinaren.c \\ om
}

Key words: UAV, Simulation Training, Matlab/Simulink, Flight Simulation, Mode Switch

\begin{abstract}
This paper elaborates the composition and function of the flight simulation system according to characteristics of UAV flight simulation in simulation training device. Flight control model and navigation model are designed based on the Matlab/Simulink to solve mode switch and other key technical difficulties in software.
\end{abstract}

\section{Introduction}

UAV has been one of important weapon equipment in modern wars and has been widely used in civil areas. As the UAV plays a more and more important role, while accelerating R\&D and equipping of advanced UAV system, countries worldwide pay more attention to research of training system and methods based on practical requirements in order to enhance UAV application performance. Currently, extensive developments and applications have been conducted for UAV simulation training systems of different types. The first aim is to improve the ability of flight personnel through simulation training. In order to implement real flight control training, a flight simulation environment should be established ${ }^{[1]}$. When the effect of flight simulation is closer to real UAV flight status, UAV control personnel's skills will be improved more.

\section{Composition of Flight Simulation System}

Flight simulation computer hardware is composed of an IPC which communicates with system equipment via network port or serial port ${ }^{[2]}$.

The software platform is based on general-purpose Windows operating system. In order to simulate the real-time control function of a stabilized turntable, Ardence RTX products will be used. This product offers a real-time subsystem on Windows platform to ensure real-time control, tracking and response on Windows platform. Software consists of two parts: flight control model and navigation model.

The former performs digital simulation of real devices of aircraft including aerodynamic device, flight-control computer, actuator, vertical gyro, rate gyro and magnetic heading sensor; the latter controls the UAV to fly at the designated flight path.

\section{Design and Implementation of Flight Simulation}

For flight control model and navigation model, the mathematical simulation method is used to simulate real devices including flight-control computer, navigation computer, aircraft power and aerodynamic systems and actuator.

Development tool. Graphical simulation modeling tool and simulation programming language are mainly used for modeling, and it is Matlab/Simulink that represents a combination of them. The modeling and debugging for the whole flight simulation including aircraft model, control law, sensor model and actuator model are implemented by Matlab/Simulink ${ }^{[3]}$. Aircraft model and sensor 
model can be selected from Simulink model library ${ }^{[4]}$. For the control law module, $\mathrm{S}$ function interfaces written in $\mathrm{C} / \mathrm{C}++$ language provided by Simulink can be used to implement mixed programming of Simulink and $\mathrm{C} / \mathrm{C}++$ language; then the model can be easily modified and debugged by calling $\mathrm{S}$ function written in $\mathrm{C} / \mathrm{C}++$ language and making good use of Simulink visual modeling capability.

Composition and function. The whole simulation model consists of flight control model (which is used to simulate dynamic characteristics of the aircraft) and navigation model. A six-DoF nonlinear model of aircraft is established based on aerodynamic data. The key to sensor model in the flight control model is how to simulate sensor noise accurately. According to physical properties of the sensor, noise signals are superposed at the output end of the sensor to simulate measured signal noise and error. Dead area, saturation and other nonlinear factors often exist in the actuator. So in the model, dead area and saturation parameters are properly set to simulate the actuator.

Flight control model. In a real UAV system, the flight control system is an integrated controller responsible for coordinating and managing all subsystems of the UAV, and is also the core of UAV flight management and control. Therefore, the implementation of the flight control module is a basic and key part in UAV flight simulation.

Mathematical modeling. To research into UAV flight control, we first have to establish a model for the research object. In modeling, the following assumptions are always made: the Earth is the inertial reference system; the aircraft is a rigid body; the weight is a constant; and acceleration of gravity does not change with flight altitude. By reference to airframe coordinates, six dynamic differential equations are established to describe the movement of aircraft. ${ }^{[5]}$ The said equations are complex in structure, so they are only suitable for numeric calculation. For the convenience of controller design, a small-disturbance linearized method can be used to obtain the small-disturbance linear state equation of UAV at the equilibrium point. ${ }^{[6]}$ Furthermore, the aircraft is symmetrical, so linear results are divided into two groups, which describe longitudinal movement and lateral movement, respectively. Therefore, five control modes are established for flight control model, including elevation holding and control, altitude holding and control, roll angle holding and control, course angle holding and control, lateral deviation control.

The longitudinal small-disturbance linear equation of UAV with wind disturbance is: ${ }^{\text {[7] }}$

$$
\dot{X}=A X+B U+F d
$$

where, $\quad\left[\begin{array}{cccc}0 & 0 & 1 & 0\end{array}\right] ; \quad[\Delta q]\left[\begin{array}{c}1 \\ 0 W_{z} / \partial x\end{array}\right] ; U_{0}$ is airspeed component along the vertical axis; $W_{x}, W_{y}$ and $W_{z}$ are three components for wind speed; $X=[\Delta V \Delta \alpha q \Delta \theta]^{T} ; U=\delta_{e}$.

The lateral small-disturbance linear equation of UAV with wind disturbance is:

$$
\dot{X}=A X+B U+F d
$$

where, $\quad\left[\begin{array}{cccc}0 & 0 & 1 & 0\end{array}\right] ; \quad\left[\begin{array}{c}\Delta \\ \Delta r\end{array}\right]\left[\begin{array}{l}\partial w_{y} / \partial z \\ \partial W_{x} / \partial z\end{array}\right] ; U_{0}$ is airspeed component along the vertical axis; $W_{x}, W_{y}$ and $W_{z}$ are three components for wind speed; $X=\left[\begin{array}{llll}\beta & p & \dot{\psi} & \gamma\end{array}\right]^{T} ; \quad U=\left[\begin{array}{ll}\delta_{a} & \delta_{r}\end{array}\right]^{T}$.

Design of control law. PID controller is used for the module. The system is under error-based negative feedback control. The controller takes the difference between system output feedback quantity and an expected value or a set value as the input quantity, and with an algorithm, obtains a control quantity to make the output quantity change with the input quantity. ${ }^{[8]}$

Take the design of longitudinal pitch channel of aircraft as an example. The pitch angle and pitch rate feedback are used for pitch attitude holding and control of the aircraft. Pitch rate feedback is realized by the angular rate compensator and the pitch angle is measured by the sensor. The throttle opening is temporarily deemed to be constant, and is not taken into account. Then, the pitch channel 
control model is designed with Simulink tool kit in Matlab.

Navigation model. The navigation system is an integral part of the UAV system. It is capable of providing support for tactical operations of the UAV through satellite navigation, AWACS guiding, ground guiding and UAV capability of detecting and tracking targets. It is mainly used to implement real-time location and automatic control of flight path of the UAV.

UAV's navigation function is based on the coordinated turn function. ${ }^{[9]}$ First, the system determines the current course of an aircraft according to voyage points, measures and calculates the lateral deviation distance between aircraft and flight path, track deviation angle and current ground velocity of aircraft in real time, and then solves a lateral driving signal in accordance with the navigation control law, and gives a bank angle to control the aircraft to enter coordinated turn, and when both lateral deviation distance and track deviation angle are zero, the aircraft performs a straight and level flight along the current flight course until it enters the next point.

The simulation of navigation control law is based on flight control law simulation. The navigation control law is designed by integrating each separate channel into full dimension simulation and taking cross track distance and yaw angle as input values.

\section{Implementation of Simulation Technology Difficulties}

The flight simulation system is very close to a real system, but different from the real system. In simulation training, UAV is required to perform some extreme actions and random switch among modes, and humanity principle shall be followed during the training. This leads to considerable difficulty and many logical problems in programming. The whole module is of purely digital analog, so mode switch in real-time module may involve problems about zero clearing of many integral terms. In case of failure in timely zero clearing, accumulated values will affect the whole flight simulation result, and even cause systematic divergence, so that the control law could not be successfully implemented. Mutual independence of longitudinal channel and lateral channel is used. When receiving a command about changing longitudinal movement, the system only clears integral terms under longitudinal control, and integral terms under lateral control will keep accumulating until a command about changing lateral movement is received. Zero clearing of lateral integral terms is performed in a similar way.

Abovementioned processes will both satisfy requirements of practical simulation training and show simulative extreme action simulation, thereby training operator's emergency response capability.

\section{Conclusion}

The flight simulation design of the simulation training device is implemented in the abovementioned method. According to the simulation result, control of modes of the aircraft meets specification requirements, the transition process during switch among modes is stable and the flight profile trend coincides. The design model can truly simulate UAV flight control and navigation and implement real-time simulation of pre-flight preparation, launch, cruising, program-control, manual control, mode switch and recovery controlled by the ground console. It has high confidence level and reliability in simulation, strong expandability and wide application.

\section{Acknowledgement}

This work is supported by the natural science foundation of Hubei Province No.2014CFB569. This work is also supported by the research project of Hubei Province Department of Education Grant No.Q20133008. 


\section{References}

[1] Peng Hua, Shen Weiqun, Song Zishan, A Real-time Management System of Flight Simulation Based on VxWorks, Journal of System Simulation, 2003, 15(7): 966-968.

[2] Zhang Ning, Chen Ning, Ji Yun, Zhu Jiang, Research on The Integrated Method of Flight Simulation System Based on A Flight Simulator, Flight Dynamics, 2010, 28(3): 39-42.

[3] Zhang Lei, Jiang Hongzhou, Qi Panguo, Li Hongren, Flight Simulation Based on Matlab, Computer Simulation, 2006, 23(6): 57-61.

[4] Shang Wenxuan, Wang He, Gao Ya, The Avionic System Platform Based on Flight Simulation of Simulink and Its Application, Electronics Optics \& Control, 2014, 21(8): 6-9.

[5] Zhang Minglian, Flight Control System, National Defense Industry Press, 1993.

[6] Xu Hailiang, Li Junyang, Fei Shumin, Design and Implementation of Digital Flight Simulation Platform, Journal of Southeast University(Natural Science Edition), 2011, 41(1): 113-117.

[7] Su Jijie, Zheng Xing, Lin Dongsheng, Yang Yi, Design and Implementation of Simulative Training System for UAV, Journal of System Simulation, 2009, 21(5): 1343-1346.

[8] Li Chao, Wang Jiangyun, Han Liang, Development of Fixed Wing Aircraft Flight Simulation System Based on Matlab, Journal of System Simulation, 2013, 25(8).

[9] Gen Tongfen, Huang Daqing, Full Process Simulation of UAV Auto-pilot Flight Based on Simulink, Aeronautical Computing Technique, 2010, 40(5): 112-116. 